\title{
Proof Formalism General Quantum Density Commutator Matrix Physics
}

\author{
Iyer $\mathbf{R}^{1 *}$ and Malaver $\mathbf{M}^{2}$ \\ ${ }^{1}$ Engineeringinc International Teknet Global Platform, USA \\ ${ }^{2}$ Department of Basic Sciences, Maritime University of the Caribbean, Venezuela
}

*Corresponding author: Rajan Iyer, Engineeringinc International Teknet Global Platform, USA, Email: engginc@msn.com

Research Article

Volume 5 Issue 2

Received Date: July 12, 2021

Published Date: August 02, 2021

DOI: $10.23880 / p s b j-16000185$

\section{Abstract}

Formalism proofing general derivation, applying matrix properties operations, showing fundamental relationships with inner product to outer product has been advanced here. This general proof formalism has direct application with physics to quantify quantum density at micro scale level to time commutator at macro scale level. System of operator algebraic equations has been rigorously derived to obtain analytic solutions which are physically acceptable. Extended physics application will include metricizing towards unitarization to achieve gaging Hamiltonian mechanics to electromagnetic gravitational strong theory, towards grand unifying physics atomistic to astrophysics or vice versa via quantum relativistic general physics thereby patching to classical physics fields energy.

Keywords: Algebra Matrix Properties; Formalism Inner Product Outer Product Relationships; Scalar Tensor General Functional Algorithm; Quantum Density Physical Time Event Commutator Quantifiability; Metricizing unitarization gage theory energy matter; Micro macro connection with quantum relativity; Atomistics to astrophysical natural phenomena

\section{Introduction}

Inner products and outer products from key aspects with matrix algebra to quantify scalar fields to the functional tensors have been utilized elsewhere thoroughly [1,2]. Matrix properties with example transpose [1], kets, bras, similar matrices key quantum physical algorithm [2] have direct applicability to Science, Engineering, Technology, and Mathematical Computing [3-5]. While local systems may have typical functional relationships, local to global linking require scalar to typical tensor fields. Examples are observables of quantum density matrix operator eigenfunctions having general energy fields manifesting time event density process matrix; especially, time fields that are typical of micro-blackholes, analytically evident from differential equations (43) and (46) formally generally developed per Iyer Markoulakis mathematical modeling abstracting physical observational measurements [4] will determine these processes. Forces that are attractive versus repulsive observable fields have been successfully solved by transforms with the ansatz Iyer Markoulakis Helmholtz Hamiltonian mechanics model generalizing these partial differential equations of entity motion states with eigen matrices to get to algebraic equations, facilitating interpretative physical analyses $[4,5]$; these have advantages of obviating singularity problems. Quantum results obtained by such fundamental formalisms of Iyer Markoulakis [4] have been applied by the first author of this paper in Malaver, et al. publications of dark energy star astrophysics [6]. We have achieved solving with interpretative physical analysis of dark energy star quantum astrophysics, with ansatz Helmholtz Hamiltonian mechanics Einstein Maxwell Gauss Bonnet gravity metric potential, anisotropy, interior energy density, and other related physical properties of such stars [6], which 
Physical Science \& Biophysics Journal

may hold key to understanding genesis of the universe with grand unification theory quantum relativistic generalizing the classical mechanics with gage physics $[7,8]$.

The aim of this paper is to generalize formalism provable solutions to link inner product to outer product such that scalars/tensors algebraic matrices are graphically relatable. System of operator algebraic equations has been rigorously derived to obtain analytic solutions which are physically acceptable. The paper is organized as follows: Section 4 presents the framework of mathematical formalism relating outer to inner product matrix generalization. Physical analyses with interpretive physics that apply results of ansatz general proof formalism are presented in Section 5. Overall summary with conclusions of proof derivation, physical analyses, potential large-scale applicability to physics with mathematical sciences, that may extend to algorithmic IT computing applications are presented further in Section 6, Sections 7 and 8 give acknowledgements and provide references.

\section{Mathematical Formalism Outer to Inner Product Matrix Generalizing}

Properties of matrix transpose [1] with $\mathbf{A}$ and $\mathbf{B}$ as matrices, and $\mathrm{c}$ as scalar are known to satisfy following general relationships:

$$
\left(\mathbf{A}^{\mathrm{T}}\right)^{\mathrm{T}}=\mathbf{A} ;(\mathbf{A}+\mathbf{B})^{\mathrm{T}}=\mathbf{A}^{\mathrm{T}}+\mathbf{B}^{\mathrm{T}} ;(\mathbf{A B})^{\mathrm{T}}=\mathbf{B}^{\mathrm{T}} \mathbf{A}^{\mathrm{T}} ;(\mathbf{c} \mathbf{A})^{\mathrm{T}}=\mathbf{c A}^{\mathrm{T}} ; \operatorname{det}\left(\mathbf{A}^{\mathrm{T}}\right)=\operatorname{det}(\mathbf{A}) ;[\mathbf{A} \cdot \mathbf{B}]=\mathbf{A}^{\mathrm{T}} \mathbf{B} ;\left(\mathbf{A}^{\mathrm{T}}\right)^{-1}=\left(\mathbf{A}^{-1}\right)^{\mathrm{T}},
$$

where superscript $\mathrm{T}$ : transpose operator, and det: determinant.

Applying these properties, to inner and outer matrix products, with arbitrary example matrices: T, $\mathbf{U}, \boldsymbol{\&} \mathbf{E}$ and operating them sequentially, utilizing matrices' concepts of bras-kets [2], we get: $(<\mathbf{A} \mid)^{\mathrm{T}}=\mid \mathbf{A}>$ or $(\mid \mathbf{A}>)^{\mathrm{T}}=<\mathbf{A} \mid$.

Let inner product $<\mathbf{E} \mid \mathbf{T}>=\mathbf{p}$, and outer product $|\mathbf{E}><\mathbf{T}|=\mathbf{q}$ (1)

We will algorithmically show that:

Outer product $(|\mathbf{E}><\mathbf{T}|)^{\mathrm{T}}=(<\mathbf{T} \mid)^{\mathrm{T}}(\mid \mathbf{E}>)^{\mathrm{T}}=|\mathbf{T}><\mathbf{E}|$ (2)

Inner Product $(<\mathbf{E} \mid \mathbf{T}>)^{\mathrm{T}}=(\mid \mathbf{T}>)^{\mathrm{T}}(<\mathbf{E} \mid)^{\mathrm{T}}=<\mathbf{T} \mid \mathbf{E}>$ (3)

Equations (1) \& (2) will give:

$(|\mathbf{E}><\mathbf{T}|)^{\mathrm{T}}=|\mathbf{T}><\mathbf{E}|=\mathbf{q}^{\mathrm{T}}(4)$

Let commutator matrix $<\mathbf{E}|\mathbf{U}| \mathbf{T}>=\mathbf{p F}$ (5)

Then operating transpose, $(<\mathbf{E}|\mathbf{U}| \mathbf{T}>)^{\mathrm{T}}=\left\langle\mathbf{T}\left|\mathbf{U}^{\mathrm{T}}\right| \mathbf{E}>=(\mathbf{p F})^{\mathrm{T}}(6)\right.$

Proof of Equation (6): Let matrix $\mathbf{A}=<\mathbf{E} \mid$, and matrix $\mathbf{B}=\mathbf{U} \mid \mathbf{T}>$. Per [1] matrix properties listed above $(\mathbf{A B})^{\mathrm{T}}=\mathbf{B}^{\mathrm{T}} \mathbf{A}^{\mathrm{T}} ;$ per [2] bras-kets concepts $(<\mathbf{A} \mid)^{\mathrm{T}}=\mid \mathbf{A}>$ or $(\mid \mathbf{A}>)^{\mathrm{T}}=<\mathbf{A} \mid$. So, we are able to write: $(<\mathbf{E} \mid(\mathbf{U} \mid \mathbf{T}>))^{\mathrm{T}}=\left\{(\mathbf{U} \mid \mathbf{T}>)^{\mathrm{T}}\right\}(<\mathbf{E} \mid)^{\mathrm{T}}$. Then, repeated transpose operations will give results that: $\left\{(\mathbf{U} \mid \mathbf{T}>)^{\mathrm{T}}\right\}(<\mathbf{E} \mid)^{\mathrm{T}}=\left\{(\mid \mathbf{T}>)^{\mathrm{T}} \mathbf{U}^{\mathrm{T}}\right\}(\mid \mathbf{E}>)=\left\{<\mathbf{T} \mid \mathbf{U}^{\mathrm{T}}\right\}(\mid \mathbf{E}>)=<\mathbf{T}\left|\mathbf{U}^{\mathrm{T}}\right| \mathbf{E}>$.

Equation (1) gives $<\mathbf{E} \mid \mathbf{T}>=\mathbf{p}$; having identity $(|\mathbf{U}| \mathbf{T}>)(\mathbf{U} \mid \mathbf{T}>)^{-1}=$ I, and Equation (5) we can get:

$<\mathbf{E}|\mathbf{T}>=<\mathbf{E}| \mathrm{I}|\mathbf{T}>=<\mathbf{E}| \mathbf{U}\left|\mathbf{T}>(\mathbf{U} \mid \mathbf{T}>)^{-1}\right| \mathbf{T}>=(\mathbf{p F})(\mathbf{U} \mid \mathbf{T}>)^{-1} \mid \mathbf{T}>$

Transposing by applying properties [1,2]: $(<\mathbf{E} \mid \mathbf{T}>)^{\mathrm{T}}=\left[(\mathbf{p F})(\mathbf{U} \mid \mathbf{T}>)^{-1} \mid \mathbf{T}>\right]^{\mathrm{T}}=\left[(\mathbf{U} \mid \mathbf{T}>)^{-1} \mid \mathbf{T}>\right]^{\mathrm{T}}(\mathbf{p F})^{\mathrm{T}}$. We show also by applying properties [1,2]: $\left[(\mathbf{U} \mid \mathbf{T}>)^{-1} \mid \mathbf{T}>\right]^{\mathrm{T}}=(\mid \mathbf{T}>)^{\mathrm{T}}\left[(\mathbf{U} \mid \mathbf{T}>)^{-1}\right] \mathbf{T}=<\mathbf{T}\left|\left[(\mathbf{U} \mid \mathbf{T}>)^{\mathrm{T}}\right]^{-1}=<\mathbf{T}\right|\left[<\mathbf{T} \mid \mathbf{U}^{\mathrm{T}}\right]^{-1}$, since $(\mathbf{A T})^{-1}=\left(\mathbf{A}^{-1}\right) \mathbf{T}$, per $[1]$, alongside other 
transpose properties. Therefore,

$(<\mathbf{E} \mid \mathbf{T}>)^{\mathrm{T}}=\left[(\mathbf{p F})(\mathbf{U} \mid \mathbf{T}>)^{-1} \mid \mathbf{T}>\right]^{\mathrm{T}}=\left[(\mathbf{U} \mid \mathbf{T}>)^{-1} \mid \mathbf{T}>\right]^{\mathrm{T}}(\mathbf{p F})^{\mathrm{T}}=<\mathbf{T} \mid\left[<\mathbf{T} \mid \mathbf{U}^{\mathrm{T}}\right]^{-1}(\mathbf{p F})^{\mathrm{T}}(7)$

Further transpose of Equation (1) will give: $(<\mathbf{E} \mid \mathbf{T}>)^{\mathrm{T}}=<\mathbf{T} \mid \mathbf{E}>=\mathbf{p}^{\mathrm{T}}(8)$

Equations (7) \& (8) will give thereby:

$\mathbf{p}^{\mathrm{T}}=<\mathbf{T} \mid\left[<\mathbf{T} \mid \mathbf{U}^{\mathrm{T}}\right]^{-1}(\mathbf{p F})^{\mathrm{T}} ; \quad$ then, multiplying equivalently both sides by $(<\mathbf{T} \mid)^{-1}$, we get:

$(<\mathbf{T} \mid)^{-1} \mathbf{p}^{\mathrm{T}}=(<\mathbf{T} \mid)^{-1}<\mathbf{T} \mid\left[<\mathbf{T} \mid \mathbf{U}^{\mathrm{T}}\right]^{-1}(\mathbf{p F})^{\mathrm{T}}=\left[<\mathbf{T} \mid \mathbf{U}^{\mathrm{T}}\right]^{-1}(\mathbf{p F})^{\mathrm{T}} ;$ multiplying $\quad$ both $\quad$ sides $\quad$ by $\quad<\mathbf{T} \mid \mathbf{U}^{\mathrm{T}}, \quad$ we get

$\left[<\mathbf{T} \mid \mathbf{U}^{\mathrm{T}}\right](<\mathbf{T} \mid)^{-1} \mathbf{p}^{\mathrm{T}}=(\mathbf{p F})^{\mathrm{T}}$ or $<\mathbf{T} \mid \mathbf{U}^{\mathrm{T}}(<\mathbf{T} \mid)^{-1} \mathbf{p}^{\mathrm{T}}=(\mathbf{p F})^{\mathrm{T}}$

Multiplying both sides with $\mid \mathrm{E}>$ we get:

$|\mathbf{E}><\mathbf{T}| \mathbf{U}^{\mathrm{T}}(<\mathbf{T} \mid)^{-1} \mathbf{p}^{\mathrm{T}}=\mid \mathbf{E}>(\mathbf{p F})^{\mathrm{T}}(9)$

Since Equation (1) gives: $|\mathbf{E}><\mathbf{T}|=\mathbf{q}$, from Equation (9) we arrive at:

$\mathbf{q} \mathbf{U}^{\mathrm{T}}(<\mathbf{T} \mid)^{-1} \mathbf{p}^{\mathrm{T}}=\mid \mathbf{E}>(\mathbf{p F})^{\mathrm{T}}$; then, multiplying equivalently both sides by identity $\mathbf{q}^{-1} \mathbf{q}=\mathrm{I}$, we get: $\mathbf{U}^{\mathrm{T}}(<\mathbf{T} \mid)^{-1} \mathbf{p}^{\mathrm{T}}=\mathbf{q}^{-1} \mid \mathbf{E}>(\mathbf{p F})^{\mathrm{T}}$; again, operating multiplication with inverses and transposes, also: Since, $\mathbf{U}^{\mathrm{T}}(<\mathbf{T} \mid)^{-1} \mathbf{p}^{\mathrm{T}}=\left\{\left[(<\mathbf{T} \mid)^{-1}\right]^{\mathrm{T}} \mathbf{U}\right\}^{\mathrm{T}} \mathbf{p}^{\mathrm{T}}=\left\{(\mid \mathbf{T}>)^{-1} \mathbf{U}\right\}^{\mathrm{T}} \mathbf{p}^{\mathrm{T}}=\left[\left\{(\mid \mathbf{T}>) \mathbf{U}^{-1}\right\}^{-1}\right]^{\mathrm{T}} \mathbf{p}^{\mathrm{T}}=\left[\left\{(\mid \mathbf{T}>) \mathbf{U}^{-1}\right\}^{\mathrm{T}}\right]^{-1} \mathbf{p}^{\mathrm{T}} \mathbf{U}^{\mathrm{T}}(<\mathbf{T} \mid)^{-1} \mathbf{p}^{\mathrm{T}}=\mathbf{q}^{-1} \mid \mathbf{E}>(\mathbf{p} \mathbf{F})^{\mathrm{T}} \quad$ will $\quad$ become $\left[\left\{(\mid \mathbf{T}>) \mathbf{U}^{-1}\right\}^{\mathrm{T}}\right]^{-1} \mathbf{p}^{\mathrm{T}}=\mathbf{q}^{-1} \mid \mathbf{E}>(\mathbf{p F})^{\mathrm{T}}$

Multiplying equivalently both sides by $\left\{(\mid \mathbf{T}>) \mathbf{U}^{-1}\right\}^{\mathrm{T}}$, and operating transposes, we get:

$\mathbf{p}^{\mathrm{T}}=\left\{(\mid \mathbf{T}>) \mathbf{U}^{-1}\right\}^{\mathrm{T}} \mathbf{q}^{-1} \mid \mathbf{E}>(\mathbf{p F})^{\mathrm{T}}=\left\{(\mid \mathbf{T}>) \mathbf{U}^{-1}\right\}^{\mathrm{T}}\left\{(\mathbf{p F})\left(\mathbf{q}^{-1} \mid \mathbf{E}>\right)^{\mathrm{T}}\right\}^{\mathrm{T}}=\left[\left\{(\mathbf{p F})\left(\mathbf{q}^{-1} \mid \mathbf{E}>\right)^{\mathrm{T}}\right\}\left\{(\mid \mathbf{T}>) \mathbf{U}^{-1}\right\}\right]^{\mathrm{T}}$

Repeating operation with transposes equivalently, $\mathbf{p}=\mathrm{pF}\left(\mathbf{q}^{-1} \mid \mathbf{E}>\right)^{\mathrm{T}} \mid \mathbf{T}>\mathbf{U}^{-1}$; applying Equations (1) and (5), we hence get the result: $\left\langle\mathbf{E}|\mathbf{T}>=<\mathbf{E}| \mathbf{U}\left|\mathbf{T}>(|\mathbf{E}><\mathbf{T}|)^{-1}\right| \mathbf{E}>\right) \mathbf{T} \mid \mathbf{T}>\mathbf{U}^{-1}(10)$

\section{Physical Analysis with Interpretive Physics}

Above formalism will allow us to consider observables that are commutable from micro to macro regime. Our example, having Markoulakis 3D observations with the Ferrolens of the Quantum Magnet field quite recently provide revelations showing that a quantum magnet physically consists of two opposite magnetic monopole charges [9]. Thus, quantum magnet will appear to have two joint counter rotating magnetic flux monopole vortices jointed together to form vortex fields of dipolar magnet in real space time [9].
Such novel mechanisms required ansatz formalism, with Iyer Markoulakis applying Helmholtz decomposition fields analysis of vortex dynamics, then gaged to electromagnetic quanta, with upper and the lower indices with the wave functions that can be used to denote the vortex actions [4]. Here, general results with matrix operations above can be applied to Iyer Markoulakis Helmholtz Hamiltonian mechanics to derive micro quantum to macro astrophysical parameters, as shown with equivalent substitutions of parameters of arbitrary matrices mathematics to specific matrices physics below.

Let $\quad \mathbf{E}=\boldsymbol{\Psi}_{\mu}(t) ; \mathbf{T}=\boldsymbol{\Psi}^{\mu}(t) ; \mathbf{U}=\mathbf{V} ;|\mathbf{E}><\mathbf{T}|=\boldsymbol{\rho}(t) ;<\mathbf{E} \mid \mathbf{T}>={ }^{\mathbf{\Psi}} \partial{ }^{\mathbf{\Psi}} \mu\left(\mathbf{E}|\mathbf{U}| \mathbf{T}>={ }^{\mathbf{\Psi} \mu} \partial^{\mathbf{\Psi} \mu} \cdot F^{E}{ }_{t}\right.$

Applying equation (10) substitution, we get result applying matrix properties [1,2]:

$\left.\boldsymbol{\Psi}_{\mu} \partial^{\boldsymbol{\Psi}_{\mu}}={ }^{\boldsymbol{\Psi}_{\mu}} \partial^{\boldsymbol{\Psi}_{\mu}} \cdot F^{E}{ }_{t}(\boldsymbol{\rho}(t))^{-1} \mid \boldsymbol{\Psi}_{\mu}(t)>\right)^{T} \mid \boldsymbol{\Psi}^{\mu}(t)>\mathbf{V}^{-\mathbf{1}}$ i.e. 
$\left.\Psi^{\mu} \partial^{\Psi_{\mu}} \cdot F^{E}(\boldsymbol{\rho}(t))^{-1} \mid \boldsymbol{\Psi}_{\mu}(t)>\right)^{T} \mid \boldsymbol{\Psi}^{\mu}(t)>\mathbf{V}^{-1}={ }^{\Psi_{\mu}} \partial^{\Psi^{\mu}}$

If $\mathrm{F}_{\mathrm{t}}^{\mathrm{E}}$ scalar functional, it will reduce to

$\left.F^{E}{ }_{t}\left[{ }^{\Psi \mu} \partial^{\Psi \mu}(\boldsymbol{\rho}(t))^{-1} \mid \boldsymbol{\Psi}_{\mu}(t)>\right)^{T} \mid \boldsymbol{\Psi}^{\mu}(t)>\mathbf{V}^{-\mathbf{1}}\right]={ }^{\boldsymbol{\Psi}^{\mu}} \partial^{\boldsymbol{\Psi}^{\mu}}$

Multiplying both sides by $\left.\left[{ }^{\Psi_{\mu}} \partial^{\Psi_{\mu}}(\boldsymbol{\rho}(t))^{-1} \mid \boldsymbol{\Psi}_{\mu}(t)>\right)^{T} \mid \boldsymbol{\Psi}^{\mu}(t)>\mathbf{V}^{-\mathbf{1}}\right]^{-1}$ we get:

$\left.F^{E}{ }_{t}={ }^{\Psi_{\mu}} \partial^{\boldsymbol{\Psi}_{\mu}}\left[{ }^{\boldsymbol{\Psi}_{\mu}} \partial^{\boldsymbol{\Psi}_{\mu}}(\boldsymbol{\rho}(t))^{-1} \mid \boldsymbol{\Psi}_{\mu}(t)>\right)^{T} \mid \boldsymbol{\Psi}^{\mu}(t)>\mathbf{V}^{-\mathbf{1}}\right]^{-1}={ }^{\boldsymbol{\Psi}_{\mu}} \partial^{\boldsymbol{\Psi}_{\mu}}\left(\boldsymbol{\Psi}^{\mu} \partial^{\boldsymbol{\Psi}_{\mu}}\right)^{-1} \boldsymbol{\rho}(t)\left(\left(\mid \boldsymbol{\Psi}_{\mu}(t)>\right)^{T}\right)^{-1}\left(\mid \boldsymbol{\Psi}^{\mu}(t)>\right)^{-1} \mathbf{V}$

On simplifying we can get:

$$
\begin{aligned}
F_{t}^{E} & =\boldsymbol{\rho}(t)\left(\left(\mid \boldsymbol{\Psi}_{\mu}(t)>\right)^{T}\right)^{-1}\left(\mid \boldsymbol{\Psi}^{\mu}(t)>\right)^{-1} \mathbf{V}=\boldsymbol{\rho}(t)\left(<\boldsymbol{\Psi}_{\mu}(t) \mid\right)^{-1}\left(\mid \boldsymbol{\Psi}^{\mu}(t)>\right)^{-1} V=\boldsymbol{\rho}(t)\left(<\boldsymbol{\Psi}_{\mu}(t) \mid \boldsymbol{\Psi}^{\mu}(t)>\right)^{-1} \mathbf{V} ; \\
F^{E} & =\boldsymbol{\rho}(t)\left(<\boldsymbol{\Psi}_{\mu}(t) \mid \boldsymbol{\Psi}^{\mu}(t)>\right)^{-1} \mathbf{V}(11)
\end{aligned}
$$

We are showing only potentially that graphing with metrix protocol [3] applicable by setting that $Y=f(X)$, with $\mathrm{X}=\rho(\mathrm{t})$ and $\mathrm{Y}=\mathrm{F}_{\mathrm{t}^{\mathrm{E}}}$; observables will show how then $\mathrm{a}$ functional commutator $[10,11], \mathrm{F}^{\mathrm{E}}$ will vary with quantum density matrix, $\rho(t)$ \{which will typically represent pure state like coupling constant with general relativity\} [12,13]. We can then interpret $f$, the function operator transforming micro to macro parametrically quantum density matrix, $\rho(t)$ to functional commutator, $\mathrm{F}^{\mathrm{E}}{ }^{\prime}$, with inner product of the wave eigenfunctions acting alongside general energy fields $[14,15]$, $\{\mathrm{V}$, the general energy field is like scalar potential in general relativity\}. Effectively, quantum density matrix, $\rho(t)$ can be seen to be influencing time event process via energy quanta. Time fields that are typical of micro-blackholes analytically is evident from differential equations (43) and (46) in Iyer Markoulis formalism [4] that determine these processes. Notable is also that outliers with X-Y plot of real data may provide observables of monopoles that may be measurable systematically with physical analysis, such as observables measured experimentally in Bose-Einstein condensates as well as within experimental measurements of monopoles using spin ice specifics $[4,5]$.

Further understanding with quantum regime that are event-based states may be linked to classical global regime that are time-based systems. As an example, linking tension between the microscopic quantum states of super-imposed possibility, and macroscopic "classical" world objective states enabling mapping some pure product effects to entangled effects are shown to have proper applicability in the quantum computing aspects [16]. The micro macro connection of quantum density to time commutator fields possibly link to knowledge of the quantum information fields [17]. Scalar potential exemplified here relate concurrently with astrophysical relativistic physics, where simply fixing the scalar potential can be systemically utilized further to obtain scalar Gauss-Bonnet coupling function [18].

Mathematical formalism generalizing inner to outer matrix product to enable establishing functional relationship among time commutator, energy matrix, eigenfunction time field's evolution up and down tensors [4,5] has been established here thoroughly. Ansatz formalism highlights only very few examples to potential physics applications, especially in the quantum field physics, relativistic astrophysics, grand unification physics theory of everything to convolve Standard Model, String Theory, SUSY, and possibility of seamlessly stitching to classical physics with enhancement gaging mechanics with electromagnetism gravity. Here, it has only been the main goal and purpose to derive formally mathematical algebra matrix that will automatically problem solve for physics, that author has also successfully demonstrated here. Expectation is that numerous areas that will include Physical Sciences, Mathematical Computing, Artificial Intelligence Expert IT, Engineering, Technology, and Economics areas will eventually find usage of this general formalism algorithm.

\section{Summary Conclusions}

Fundamental formalism mathematics borne out of basic matrix properties operating on inner product and outer product relationships generalizing scalar tensor micro macro connectivity quantifiability physics has been achieved here.

Physical mathematical gaging mechanics to electromagnetic theory has gotten facilitated by ansatz formalism able to pull out observables with parameters of quantum density matrix operator eigenfunctions having general energy fields, $\mathrm{E}$, functional commutator, $\mathrm{F}_{\mathrm{t}}^{\mathrm{E}}$. with density matrix, $\rho(t)$, influencing time event process. Time fields that are typical of micro-blackholes have been shown 
to be analytically evidenceable from differential equations will determine these processes.

Authors hope that general formalism derived here can be applied to pull out observables of monopoles with parameters that may be measurable systematically with physical analysis, such as observables measured experimentally in Bose-Einstein condensates as well as within experimental measurements of monopoles using spin ice specifics, providing an independent means of quantifying physical phenomenological processes.

What is achieved here may be a monumental quantum leap in mathematical matrix physical algebra. We hope that mathematical matrix formalism fundamentally derived here may help in linking microscopic phenomena with macroscopic phenomena such as grand unifying physics atomistic to astrophysics or vice versa via quantum relativistic general physics thereby patching to classical physics fields energy. We are working with quantum astrophysical groups to apply this to advanced levels of gaging, grand unifying, and formulating algorithms that will stitch mathematics with physics observables, measurements, and phenomenological processes.

\section{Acknowledgements}

Encouragements with group projects support of Emmanouil Markoulakis of Hellenic Mediterranean University in Greece, and perpetual project support of Engineeringinc International Operational Teknet Earth Global Platform and Maritime University of the Caribbean in Venezuela collaborations would be appreciated highly.

\section{References}

1. Cayley A (1858) A memoir on the theory of matrices. Philosophical Transactions 148: 17-37.

2. Greensite J (2017) Bras and kets, and canonical quantization. In: Greensite J (Ed.), An Introduction to Quantum Theory. IOP Publishing, pp: 23-1 to 23-28.

3. Iyer R (2016) Applying IPZ Model Principle to Equation of Motion Mathematical Physics. Engineeringinc International Teknet Global Platform World University, USA.

4. Iyer R, Markoulakis E (2021) Theory of superluminous vacuum quanta as the fabric of Space. Phys Astron Int J 5(2): 43-53.
5. Iyer R (2021) Problem Solving Vacuum Quanta Fields. International Journal of Research and Reviews in Applied Sciences 47(1): 15-25.

6. Malaver M, Kasmaei HD, Iyer R, Sadhukhan S, Kar A (2021) A theoretical model of Dark Energy Stars in Einstein-Gauss-Bonnet Gravity. General Relativity and Quantum Cosmology, pp: 26.

7. Siegel W (1999) Fields. High Energy Physics, pp: 885.

8. $\quad$ Langacker P (2010) The standard model and beyond. $1^{\text {st }}$ (Edn.), Taylor and Francis, 7(10): 11419.

9. Markoulakis E, Konstantaras A, Chatzakis J, Iyer R, Antonidakis E (2019) Real time observation of a stationary magneton. Results in Physics 15: 102793.

10. Crewther RJ (1995) Introduction to Quantum Field Theory. High Energy Physics, arXiv, pp: 47.

11. McCoy NH (1929) On commutation formulas in the algebra of quantum mechanics. Transactions of the American Mathematical Society 31(4): 793-806.

12. Fano U (1957) Description of States in Quantum Mechanics by Density Matrix and Operator Techniques. Reviews of Modern Physics 29(1): 74-93.

13. Belinfante FJ (1980) Density matrix formulation of quantum theory and its physical interpretation. International Journal of Quantum Chemistry 17(1): 1-24.

14. Zamolodchikov AB (1979) Factorized S-matrices in two dimensions as the exact solutions of certain relativistic quantum field theory models. Annals of Physics 120(2): 253.

15. Fiderer LJ, Tufarelli T, Piano S, Adesso G (2021) General Expressions for the Quantum Fisher Information Matrix with Applications to Discrete Quantum Imaging. PRX QUANTUM 2(2): 020308.

16. Baldijão RD, Krumm M, Garner AJP, Müller MP (2020) Quantum Darwinism and the spreading of classical information in non-classical theories. Quantum Physics, arXiv, pp: 18.

17. Zizzi P (2020) Quantum Information Hidden in Quantum Fields. Quantum Reports 2(3): 459-490.

18. Odintsov SD, Oikonomou VK, Fronimos FP (2020) Rectifying Einstein-Gauss-Bonnet inflation in view of GW170817. Nuclear Physics B 958. 